\title{
Exposure to Descriptions of Traumatic Events Narrows One's Concept of Trauma
}

Payton J. Jones, M.A.

David Levari, Ph.D.

Benjamin W. Bellet, M.S.

Richard J. McNally, Ph.D.

\author{
Harvard University
}




\begin{abstract}
The word 'trauma' was originally used by psychiatrists to describe horrific events such as rape and torture that characteristically provoke extreme emotional distress. Both colloquially and clinically, the concept of psychological trauma has broadened considerably. Although many clinical scientists have expressed concern about the broadening of the concept of trauma, it remains unclear how and why this concept expansion occurs. We present two experiments in which US adults $(N=276,267)$ sequentially classified descriptions of events (e.g., "broke a leg in a bicycle accident") as either 'trauma' or 'not trauma'. In the first experiment, we manipulated the frequency of serious and nonserious events. In the second experiment, we manipulated the total range of events (i.e., participants viewed only nonserious or only serious events). Together, the experiments suggest that participants' frame of reference for events (i.e., the perceived range of event seriousness) plays a relatively more important role.

Keywords: trauma, concept creep, PTSD
\end{abstract}




\section{Exposure to Descriptions of Traumatic Events}

\section{Narrows One's Concept of Trauma}

Trauma centers treat injuries resulting from sudden physical insults to the body.

Psychological trauma is a metaphorical extension of the medical term applied to emotional harm, formalized in the appearance of posttraumatic stress disorder (PTSD) in the third edition of the Diagnostic and Statistical Manual of Mental Disorders (DSM-III; American Psychiatric Association [APA], 1980). The diagnosis emerged in part due to discussions surrounding "postVietnam war syndrome" (Shatan, 1972). The psychological complications of war veterans were grouped with those of individuals who were traumatized by rape, natural disasters, or events such as the Holocaust to form the category of PTSD (McNally, 2003). Hence, the original formulation presumed that PTSD could only arise following exposure to terrifying, presumably rare events falling outside the boundary of ordinary experience. Yet the concept of trauma has increasingly expanded to embrace a wider range of stressful events (McNally, 2016). For example, individuals have reported PTSD symptoms resulting from being bullied at work (Matthiesen \& Einarsen, 2004) or giving birth to a healthy baby after an uncomplicated delivery (Olde, van der Hart, Kleber, \& Van Son, 2006).

This raises an important question for diagnosis, treatment, and related policies: what is trauma? From a diagnostic standpoint, the current edition of the DSM (DSM-5) maintains the relatively strict definition of "exposure to actual or threatened death, serious injury, or sexual violence" (APA, 2013, p. 271). Yet among laypersons, 'trauma' often has a much broader meaning. Media outlets have applied it to include experiencing microaggressions (Williams, 2015), reading the news (Jacobs, 2018), or learning secondhand about "difficult or disturbing stories" (Lees, 2018, para. 3). 
Expansions in the usage of the term 'trauma' over time may indicate "conceptual bracket creep in the definition of trauma" (McNally, 2003, p. 231; Haslam, 2016). Concept creep refers to the expansion of a semantic boundary over time. Psychologists vary in their views on the benefits and costs of expanding the definition of trauma. On one hand, such expansions may reflect a development in deeper empathy towards individuals who suffer from various types of negative events (Haslam, 2016). In addition, concept expansion may reduce uncertainty about the (un)acceptability of certain behaviors, empowering victims and third-party allies to take more decisive action (Cikara, 2016).

On the other hand, some scholars worry that expansions in the concept of trauma dilute the meaning of the term (Haslam, 2016; McNally, 2016). In the words of the historian of military psychiatry, Ben Shephard, "Any unit of classification that simultaneously encompasses the experience of surviving Auschwitz and that of being told rude jokes at work must, by any reasonable lay standard, be a nonsense, a patent absurdity" (Shephard, 2004, p. 57).

From a sociocultural standpoint, Haslam (2016) worried that diluting the public's perception of the trauma concept might "[risk] reducing the range of people who see themselves as capable of moral agency," therefore increasing "a tendency for more and more people to see themselves as victims who are defined by their suffering, vulnerability, and innocence, and who have diminished agency to overcome their plight." From a clinical standpoint, McNally (2009) wondered whether it might alter the etiological understanding of PTSD, "[undermining] the very rationale for having a diagnosis of PTSD in the first place," potentially resulting in the misapplication of trauma treatments and the overmedicalization of normal stress, undercutting natural resilience. 
Regardless of whether concept creep is helpful or unhelpful, little is understood about how and why it occurs. One possibility is that concept expansions occur as a result of altered frequencies of exposure. For example, if overt violence occurs at a low frequency, individuals may shift their concept boundaries to encompass additional examples (e.g., classifying hateful speech as violence). Indeed, in a series of experiments, Levari and his colleagues (2018) found that shifting the prevalence of displayed categories reliably alters conceptual boundaries across several sets of stimuli and conditions. For example, when participants were shown decreasing amounts of threatening faces (relative to non-threatening faces), they expanded the range of faces they classified as threatening. The researchers dub this effect "prevalence-induced concept change", referring to the semantic shift that occurs due to changes in the relative prevalence of instances of certain categories.

Another possibility is that concept expansions may occur as a result of an altered range of experience. For instance, experiencing an event on the extreme end of a continuum (e.g., surviving a concentration camp) seems likely to have lasting effect on an individuals' conceptual boundaries, even if that event only occurs once (i.e., has a low frequency).

We attempt to test the influence of both event frequency and the total range of events as they relate to binary judgments of events as either 'trauma' or 'not trauma.' In the first study, we conducted a preregistered extension of Levari and colleagues' (2018) experiments. Specifically, we asked participants to classify events as either trauma or not trauma while manipulating the relative frequency of serious and nonserious events. In the second study, we repeated a similar experiment, but manipulated the range of events' seriousness while maintaining the relative frequencies as a constant.

\section{Experiment 1}




\section{Method}

Stimuli Generation \& Norming. We generated 600 descriptions of events covering the entire spectrum from "not at all traumatic" to "extremely traumatic." The descriptions ranged in length from 2-16 words and varied widely in their thematic and emotional content (e.g., "walked up a flight of stairs," "killed a child pedestrian while driving").

To obtain initial objective ratings of the stimuli, we conducted a pretest. We randomly divided the stimuli into six equal sets of 110 items. In each set, 98 items were unique to the set, whereas the other 12 items appeared in all sets, serving as a consistency check. We presented each set of descriptions in random order to participants recruited from Amazon Mechanical Turk (MTurk) $\left(n_{\text {total }}=250, n_{\text {set }} \approx 42\right)$. Participants were asked to rate each description on a 7-point Likert scale from "Not at all traumatic" to "Extremely traumatic". Interrater reliability on the consistent set of 12 items was good when assessed across each of 250 participants as separate judges $\left(\mathrm{ICC}_{1}=0.70 ;\right.$ Shrout $\&$ Fleiss, 1979), and excellent when considering the average value across each of the six sets $\left(\mathrm{ICC}_{1}=0.99\right)$. Inclusion and exclusion criteria for pilot raters were the same as for the main experiments, as detailed below. Pilot raters were ineligible to participate in the experiments.

Procedure. We preregistered Experiment 1 on the Open Science Framework (osf.io/tw92r). Participants were recruited from MTurk. They were allowed to participate in the study if they were adult United States residents and had an MTurk approval rate of 95\% or greater. Participants first completed a CAPTCHA and US residency screener (e.g., "What emergency number is most common in the United States?") and were immediately excluded if 
they failed either task. Participants were first given basic instructions regarding the survey ${ }^{1}$. They were then shown each event in sequence in a standardized window and were instructed to press one of two keys to indicate either "trauma" or "not trauma" for each description. They viewed each item for a minimum of 1.5 seconds before clicking and were instructed to take a break every 30 items (<5 seconds). Attention checks were interspersed throughout this task (i.e., "please press the p key on your keyboard"). After rating all items for their condition, participants completed a demographics and psychiatric history questionnaire and a human participant verifier (writing three sentences about the past weekend). They were then shown a debriefing form explaining the purpose of the experiment. As preregistered, participants were excluded from the analysis if they incorrectly answered attention checks or failed the human participant verifier. This left a total of 276 participants.

Using the rating data from the pretest, we sorted items into categories depending on their mean rating on the 7-point Likert scale in the pretest: nonserious events (mean $=1-2$ ), ambiguous events $($ mean $=3-4)$, serious events (mean $=5-7)$. We intentionally selected items that had acceptably low standard deviations in the pretest $(s d<1.6)$ to avoid selecting items that were inconsistently interpreted by different participants. We then selected 342 descriptions of events that corresponded to nonserious events (142 descriptions, e.g., "walked up a flight of stairs"), ambiguous events (100 descriptions, e.g., "broke an ankle while running") or serious events (100 descriptions, e.g., "killed a child pedestrian while driving") to be used in the main study. The exact number of items in each category was determined by a calculation that ensured we would have sufficient unique stimuli in each category given our experimental design.

\footnotetext{
1 "Some events that happen in life may be considered to be trauma. In this survey, you will read a series of [X] descriptions of events that may occur in a person's life. For each description, you will be asked to decide whether the event itself is "trauma" or "not trauma". There are no right or wrong answers."
} 
We will refer to the probability that participants were shown an item from the "serious" category in each block as the signal prevalence. For participants in the control ("stable") condition, participants were shown serious events with a signal prevalence of $33.3 \%$ throughout the experiment. For participants in the experimental ("decreasing") condition, we modified the signal prevalence over time. The signal prevalence was set at $33.3 \%$ for the first 100 trials, $16.6 \%$ for the next 50 trials, $8.3 \%$ for the next 50 trials, and $4.12 \%$ for the last 50 trials $^{2}$.

Analysis. Data were analyzed in R (R Core Team, 2019). We fit a binomial generalized linear mixed effects model to the data. In each experiment, the dependent variable was the participants' identification of a stimulus as "trauma" or "not trauma." Random effects were added for the (a) intercepts for participants and (b) slopes for trial number if they improved model fit as determined by a $\chi^{2}$ test.

Based on the results of Levari et al (2018), we predicted that individuals in the stable condition would remain consistent in their ratings over time, whereas individuals in the decreasing condition would become more lenient in their concept of trauma (i.e., have a higher likelihood of rating nonserious or ambiguous events as trauma in later trials). The predictor variables in our binomial generalized linear mixed effects model were experimental condition, pilot ratings of descriptions (i.e., "objective seriousness" of each description), trial number, and their interactions. We expected a significant three-way interaction of the predictor variables, indicating that individuals in the decreasing condition would classify nonserious or ambiguous items as "trauma" at a higher likelihood compared to individuals in the stable condition, but only at later trials.

\section{Results}

\footnotetext{
${ }^{2}$ Due to an error in the item selection algorithm, the last 25 trials showed items at an incorrect signal prevalence. These final 25 trials were therefore removed in all reported analyses for Experiment 1.
} 
A model including both random effects for the intercepts of participants and slopes for trial number showed superior fit compared to models excluding either of these effects, as determined by a $\chi^{2}$ test $(p<0.001, N=276)$. Our prediction of a three-way interaction between condition, objective seriousness of each item, and trial number was not supported $(\beta=-0.21, z=$ $-0.35, p=0.73)$. Instead, participants became stricter in their threshold of assigning the descriptor of "trauma" over time across both conditions. This effect is visualized in Figure 1. Over increasing trial numbers, the threshold shifted to the right (increasingly strict) in both conditions. Because the three-way interaction was not supported, we subsequently tested for twoway interactions, as preregistered. In the model without the three-way interaction, we found significant two-way interactions between trial number and objective seriousness, and between condition and objective seriousness, neither of which was relevant to our hypotheses, nor to the shift right across conditions apparent in the graph $(\beta=1.20,-1.69, z=3.67,-8.97$, ps $<0.001)$. However, there was no two-way interaction between trial number and condition $(\beta=0.10, z=$ $0.53, p=0.59)$, indicating that any shifting of the thresholds across trial number did not differ by condition. To ensure that this two-way interaction model was appropriate, we tested for differences in fit between it and the three-way interaction model. There was no significant difference in fit between this model and the model including the three-way interaction $(p=0.73)$.

In an exploratory model including only main effects, we indeed observed a main effect of trial number $(\beta=-0.81, z=0-8.78, p<0.001)$, which corresponds to the shift observed in the graph. As noted earlier, the effect of trial number was not moderated by condition when two-way interactions were included. We were surprised to see movement of participants' rating threshold within the stable condition, as this effect was not present in any of the seven original experiments presented by Levari et al (2018). Overall, these results did not provide support for prevalence- 
154 induced concept change in the rating of descriptions of traumatic events. Instead, they seemed to 155 indicate the presence of another, unexpected effect of narrowing across both conditions. 
Figure 1. No Effect of Prevalence, but an Effect of Trial Number on Trauma Ratings

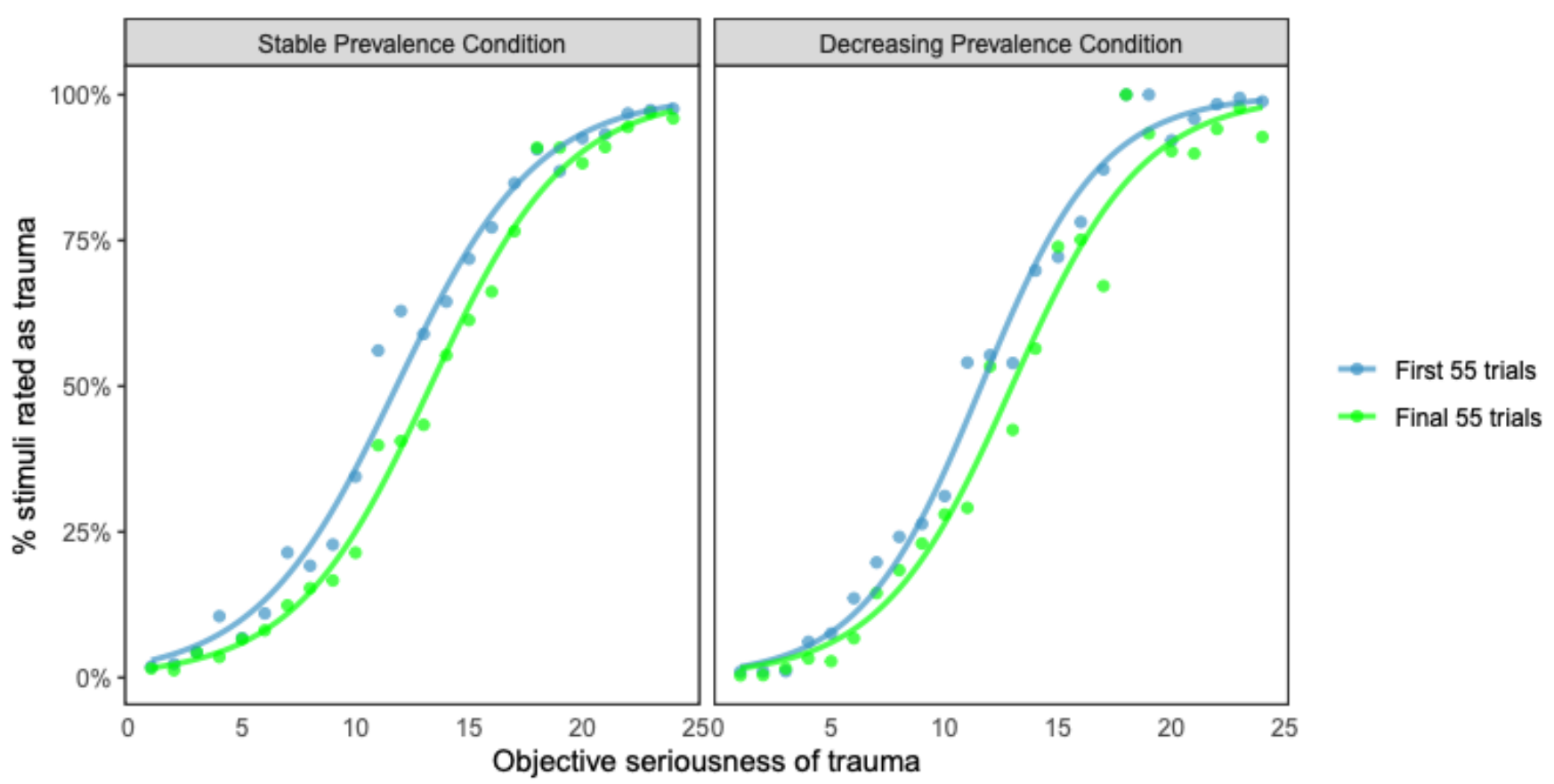

Each dot represents an event description, with lines drawn to indicate the threshold at which participants on average made the binary split between "not trauma" or "trauma" based on objective seriousness. Participants in both conditions rated descriptions more strictly in later trials compared to earlier trials. 


\section{Discussion}

In Experiment 1, we found an unexpected effect. Regardless of the prevalence of serious traumas changed over time, participants became increasingly strict in what they classified as a trauma. After rating the first several items, participants became stricter, assigning the label of "trauma" only to relatively severe events. This effect appeared strongest for the first few trials, with a gradual but smaller shift continuing in later trials (see Supplemental Figure S1).

What might explain this effect? One possibility concerns the initial range in participants' working concepts of trauma, or what Parducci (1965, p. 407) called an "implicit frame of reference." Some of our more serious trauma items included "was tortured as a prisoner of war" and "was raped by a close friend". When our sample of relatively young American participants initially considered the term trauma, such events may not readily be brought to mind. In other words, it is possible that participants saw descriptions in the experiment that were more severe than they expected to see.

We therefore tested whether the narrowing effect we observed in Study 1 was attributable to participants' frame of reference. We thus devised a new experiment with experimental conditions that directly addressed the range of events shown to participants.

\section{Experiment 2}

\section{Methods}

Our participant recruitment and data collection methods were the same as in Experiment 1, but we modified the conditions to test our hypothesis about the range of events. After exclusion, 267 participants remained. In the first condition (hereafter "nonserious range" condition), participants were shown only items decisively judged to be nontraumatic in the stimuli norming pretest (range of mean: 1-3). To illustrate, the least serious event in the 
nonserious range was "walked up a flight of stairs" and the most serious was "was not hired after a job interview." In the second condition (hereafter "serious range" condition), participants were shown only items decisively judged to be in the range of serious traumatic events (range of mean: 5-7). The least serious event in the serious range was "received chemotherapy" and the most serious was "was raped by a family member". If our hypothesis concerning the effect of the range of events was correct, we expected to see significantly stricter ratings in the high-range condition compared to the low-range condition.

To analyze the data, we used a binomial generalized linear mixed effects model. Our dependent variable was participants' binary rating of each stimulus as "trauma" or "not trauma". The predictor variables were experimental condition, pilot ratings of descriptions (i.e., "objective seriousness" of each description), and trial number. In this case, we did not model a three-way interaction, as we did not expect the objective seriousness of trauma to interact with the trial number and the condition. Instead, we hypothesized a significant two-way interaction of the condition and trial number, indicating that individuals in the serious range condition would classify items as "trauma" at a lower likelihood compared to individuals in the low-range condition, but primarily at later trials. Our model included the main effects and the hypothesized interaction term.

\section{Results}

Our prediction of a significant two-way interaction of the variables was not supported $(\beta$ $=0.22, z=0.84, p=0.40, N=267)$. Instead, our prediction that individuals in the high-range condition would classify items as "trauma" at a lower likelihood was supported, but this effect was not moderated by trial number (i.e., the effect manifested almost immediately). This is reflected by the main effect of condition on participant ratings, which remained significant 
regardless of whether the nonsignificant interaction term was in the model $(\beta=5.58 .5 .66, z=$ 17.79, 19.243, ps <0.001; see Figure 2). 
Figure 2. Range Restrictions in Ratings of Trauma

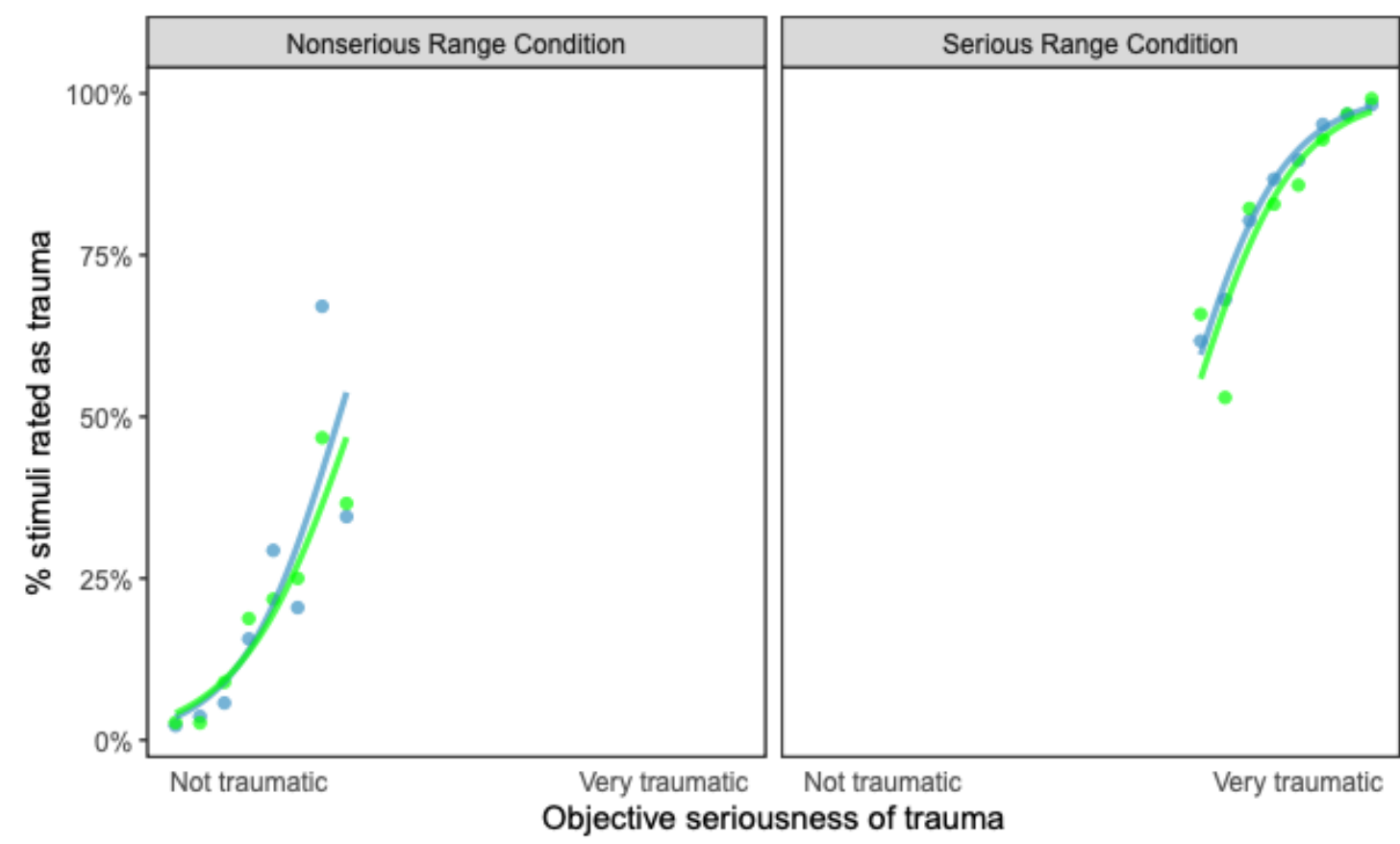

- Initial 30 trials

- Final 30 trials

Each dot represents an event description, with lines drawn to indicate the threshold at which participants on average made the binary split between "not trauma" or "trauma" based on objective seriousness. Controlling for the objective seriousness of the descriptions, participants who saw only serious events were much stricter than expected compared to participants who saw only nonserious events. 
To interpret the results, it is useful to consider them in relation to the Experiment 1 data. In the stable condition of Experiment 1, participants were shown events from the full range in random order. Because there was no manipulation of frequency, this condition is conceptually identical to the two Experiment 2 conditions. We can therefore use these data to visualize a hypothetical "full range" condition. Keep in mind that this visualization is intended for explanatory purposes, not for inference.

If the range hypothesis were correct, we would expect participants in the "nonserious range" condition to be the most lenient, followed by participants in the proxy "full range" condition, with individuals in the "serious range" condition behaving the most strictly. Indeed, this pattern applies, clearly visible in Figure 3. Individuals in the nonserious range condition have the most leftward curve, indicating that even mild items were frequently classified as traumatic. In contrast, individuals in the serious range condition had the curve farthest to the right, indicating that even serious descriptions were often classified as nontraumatic. The proxy full range condition falls between these two. Recall that when separated by time, the early trials of the full range condition produced a curve farther to the left, whereas the later trials produced a curve farther to the right. 
Figure 3. The Influence of Range on Trauma Ratings

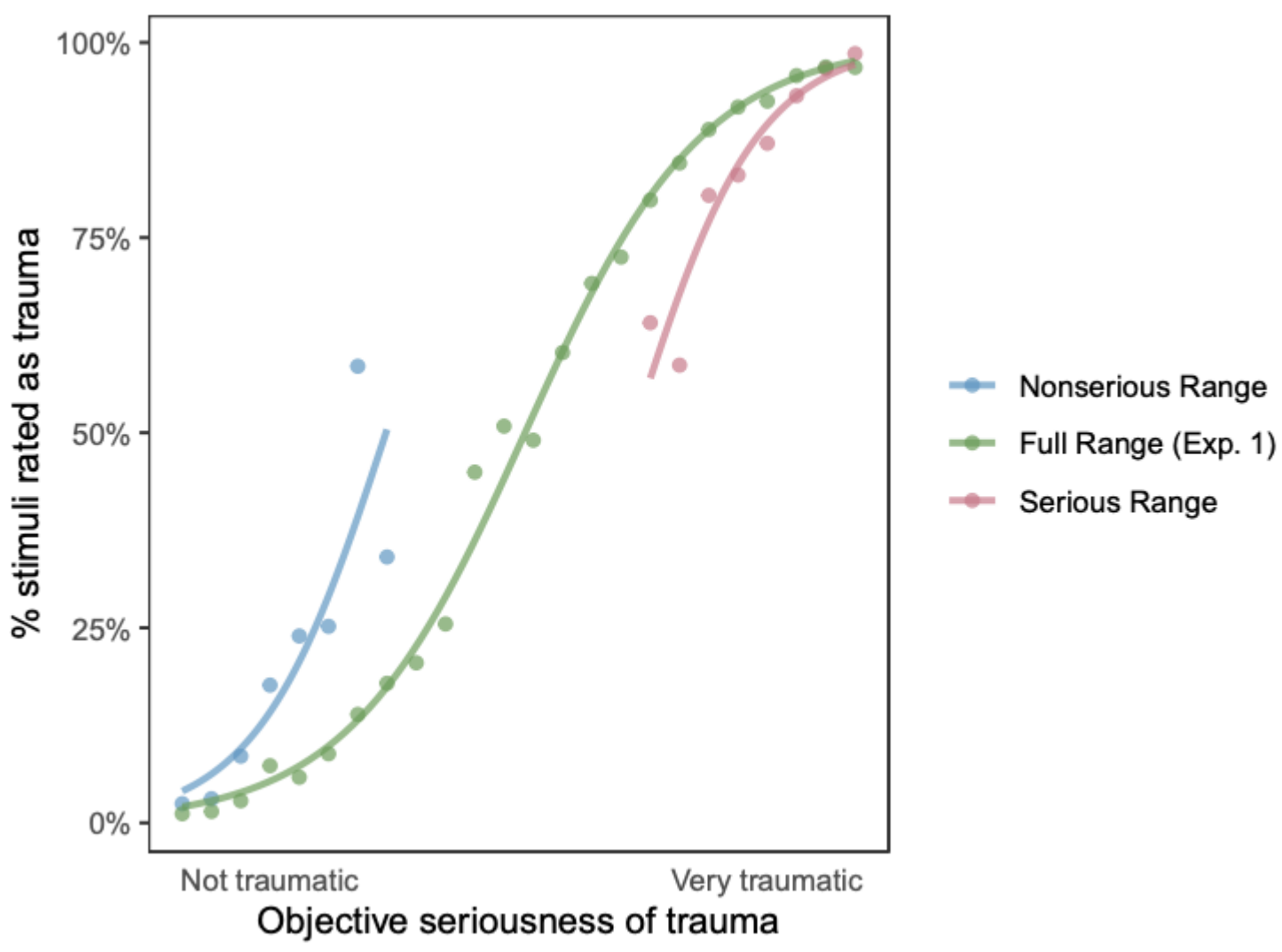

Each dot represents an event description, with lines drawn to indicate the threshold at which participants on average made the binary split between "not trauma" or "trauma" based on objective seriousness. Participants had the strictest threshold (e.g., only very serious events are "trauma") when they saw only serious events, and the most lenient threshold (e.g., even some minor events are "trauma") when they saw only nonserious events. 


\section{General Discussion}

Concepts can expand over time to include events hitherto deemed to fall outside their original boundaries. In our first experiment, we sought to determine whether the concept of trauma would expand as the most serious instances of trauma became increasingly rare. We did not find evidence of prevalence-induced concept change for trauma. Instead, we found an unexpected effect whereby individuals became stricter in their trauma ratings over time, regardless of altered frequencies.

Perhaps people underweight the role of frequency when evaluating potentially dangerous events. For example, many people dread shark attacks despite their rarity (likely an adaptive trait). Thus, exposure to serious events may affect trauma concepts even when exceedingly rare. We hypothesized that exposure to our most extreme examples broadened participants' implicit range of events.

We tested this range hypothesis in our second experiment. Indeed, we found that altering the range of events shown to participants influenced their ratings of trauma. Participants who saw only nonserious events were lenient in classifying events as trauma compared to those who saw serious events. A shift in the working range of events thus provides one plausible explanation for the narrowing effect we observed in the first experiment.

What implications does this have for the concept creep of "trauma"? Our results suggest that frames of reference shape individual judgements about the breadth of the trauma concept. This may explain seemingly paradoxical trends in the prevalence of trauma and PTSD. Epidemiological studies often indicate very high self-reported rates of exposure to trauma in first-world countries, despite relatively low rates of violence and disaster in those same countries. In one remarkable example, the lifetime rate of exposure to trauma in Canada was 
recorded as slightly higher than the rate in South Africa (76\% vs. 74\%; Dückers, Alisic, \& Brewin, 2016) ${ }^{3}$.

Furthermore, countries with high vulnerability indices (e.g., high income inequality, political corruption), such as Mexico and South Africa, have lower rates of PTSD following trauma compared to countries with lower vulnerability indices, such as Canada and the United States (Dückers et al., 2016). In the United States, rates of violent crimes such as rape steadily declined between the early 1990s and 2010s (Pinker, 2011). Yet during the same timeframe, rates of PTSD have remained relatively stable (Kessler, Sonnega, Bromet, Hughes, \& Nelson, 1995; Kessler, Berglund, Demler, Jin, Merikangas, \& Walters, 2005; Kilpatrick, Resnick, Milanak, Miller, Keyes, \& Friedman, 2013).

Our results help explain why the same stressors may be perceived as being more traumatic in an advantaged society. This is not only an epidemiological issue, but a clinical one. Negatively appraising an adverse event forms the basis for developing PTSD in the cognitive model (Ehlers \& Clark, 2000). Negative appraisals of an event incrementally predict long-term PTSD rates above and beyond other risk factors (e.g., Bryant \& Guthrie, 2005; Ponnamperuma \& Nicolson, 2016). The individual who classifies an event as a 'trauma' may be more likely to see the event through the lens of permanent harm, importance to life narrative, and risk for PTSD. This type of appraisal may promote rumination about the event and increase vulnerability (Berntsen \& Rubin, 2007).

Trauma concepts are also relevant to therapists. A therapist who works in the criminal justice system may be less likely to categorize a given event as a "Criterion A Trauma"

\footnotetext{
${ }^{3}$ An alternative explanation is that trauma is more evenly distributed in Canada, with individuals in South Africa experiencing a much greater number of traumas per person, but with both countries having a similar number of individuals never experiencing trauma.
} 
compared to a therapist who works primarily with the worried-well. Indeed, manipulations of immediate context have previously been shown to alter standardized ratings of psychopathology, a problem insufficiently addressed in clinical practice (Wedell, Parducci, \& Lane, 1990). In the broader political and educational landscape, our results suggest that reminders of very severe events may curb categorization of relatively minor events as 'trauma'.

Our research has several limitations. Although manipulating the range seemed to have the most prominent effect in our experiments, it remains unclear whether there exist conditions under which manipulation of the frequency would affect ratings of trauma. It is possible that changes in frequency do affect rating thresholds, but only over a much longer time period. Conversely, it is possible that the effects we obtained in this short timeframe are not durable. Indeed, the fact that ratings can be so easily manipulated suggests that immediate context is relatively powerful, and thus any interventions that influence trauma concepts may be overridden in future contexts. This study only provides information about exposure to descriptions of events, not direct exposure to events. It seems likely that direct exposure to events might influence trauma concepts more drastically or more durably. Future studies might explore the effect of exposure to events through audio, video, or virtual reality.

The concept of psychological trauma has expanded rapidly in the socio-political arena as well as in psychiatric diagnosis. We investigated whether a paradigm assessing prevalenceinduced concept change could help explain this concept creep. Our results indicated that unlike stimuli tested in previous experiments, such as ratings of color, threateningness, or ethicality (Levari et al., 2018), the categorization of trauma was not significantly affected by changes in prevalence. Instead, when individuals were shown the full range of events, they became stricter over time in their willingness to categorize events as trauma. A second experiment revealed that 
manipulating the range of events altered trauma ratings: individuals shown mostly benign events were lenient in categorizing events as trauma, whereas individuals shown mostly serious events were more restrained. Thus, expansions in the concept of trauma may occur primarily in frames of reference in which very serious events are absent. 


\section{References}

American Psychiatric Association. (2013). Diagnostic and statistical manual of mental disorders (5th ed.). Arlington, VA: American Psychiatric Publishing

Berntsen, D., \& Rubin, D. C. (2007). When a trauma becomes a key to identity: Enhanced integration of trauma memories predicts posttraumatic stress disorder symptoms. Applied Cognitive Psychology, 21, 417-431.

Bryant, R. A., \& Guthrie, R. M. (2005). Maladaptive appraisals as a risk factor for posttraumatic stress: A study of trainee firefighters. Psychological Science, 16, 749-752.

Cikara, M. (2016). Concept expansion as a source of empowerment. Psychological Inquiry, 27, 29-33.

Dückers, M. L., Alisic, E., \& Brewin, C. R. (2016). A vulnerability paradox in the cross-national prevalence of post-traumatic stress disorder. British Journal of Psychiatry, 209, 300-305.

Ehlers, A., \& Clark, D. M. (2000). A cognitive model of posttraumatic stress disorder. Behaviour Research and Therapy, 38, 319-345.

Lees, A. B. (2018). Yes, you can be traumatized by the media!. Psychology Today. Retrieved from https://www.psychologytoday.com/us/blog/surviving-thriving/201810/yes-you-canbe-traumatized-the-media

Jacobs, J. (2018). When the news itself is a form of trauma. New York Times. Retrieved from https://www.nytimes.com/2018/09/26/us/metoo-survivors-kavanaugh-cosby.html

Haslam, N. (2016). Concept creep: Psychology's expanding concepts of harm and pathology. Psychological Inquiry, 27, 1-17. 
Kessler, R. C., Berglund, P., Demler, O., Jin, R., Merikangas, K. R., \& Walters, E. E. (2005). Lifetime prevalence and age-of-onset distributions of DSM-IV disorders in the National Comorbidity Survey Replication. Archives of General Psychiatry, 62, 593-602.

Kessler, R. C., Sonnega, A., Bromet, E., Hughes, M., \& Nelson, C. B. (1995). Posttraumatic stress disorder in the National Comorbidity Survey. Archives of General Psychiatry, 52, 1048-1060.

Kilpatrick, D. G., Resnick, H. S., Milanak, M. E., Miller, M. W., Keyes, K. M., \& Friedman, M. J. (2013). National estimates of exposure to traumatic events and PTSD prevalence using DSM-IV and DSM-5 criteria. Journal of Traumatic Stress, 26, 537-547.

McNally, R. J. (2003). Progress and controversy in the study of posttraumatic stress disorder. Annual Review of Psychology, 54, 229-252.

McNally, R. J. (2009). Can we fix PTSD in DSM-V? Depression and Anxiety, 26, 597-600.

McNally, R. J. (2016). The expanding empire of psychopathology: The case of PTSD. Psychological Inquiry, 27, 46-49.

Olde, E., van der Hart, O., Kleber, R., \& Van Son, M. (2006). Posttraumatic stress following childbirth: a review. Clinical Psychology Review, 26, 1-16.

Parducci, A. (1965). Category judgment: a range-frequency model. Psychological Review, 72, 407-418.

Pinker, S. (2011). The better angels of our nature: Why violence has declined. New York, NY: Viking.

Ponnamperuma, T., \& Nicolson, N. A. (2016). Negative trauma appraisals and PTSD symptoms in Sri Lankan adolescents. Journal of Abnormal Child Psychology, 44, 245-255. 
R Core Team (2019). R: A language and environment for statistical computing. $R$ Foundation for Statistical Computing, Vienna, Austria. http://www.R-project.org/

Shatan, C. F. (1973). The grief of soldiers: Vietnam combat veterans' self-help movement. American Journal of Orthopsychiatry, 43, 640-653.

Shephard, B. (2004). Risk factors and PTSD: A historian's perspective. In G. M. Rosen (Ed.), Posttraumatic stress disorder: Issues and controversies (pp. 39-61). Chichester, England: John Wiley \& Sons.

Wedell, D. H., Parducci, A., \& Lane, M. (1990). Reducing the dependence of clinical judgment on the immediate context: Effects of number of categories and type of anchors. Journal of Personality and Social Psychology, 58, 319-329.

Williams, M. T. (2015). The link between racism and PTSD. Psychology Today. Retrieved from https://www.psychologytoday.com/us/blog/culturally-speaking/201509/the-link-betweenracism-and-ptsd 